\title{
$539-90$

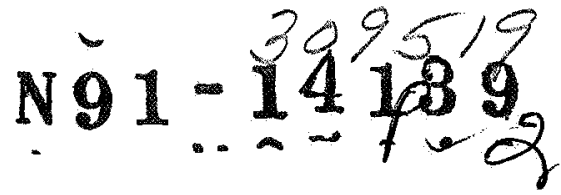

\section{A MODEL FOR THE INFRARED EMISSION FROM AN OB STAR CLUSTER ENVIRONMENT}

D. Leisawitz

NASA/Goddard Space Flight Center

Laboratory for Astronomy and Solar Physics

We have developed an interactive radiative transfer code that

predicts the infrared emission from an $H$ II region containing diffuse ionized and atomic gas and dense molecular clouds. This model complements our investigation of the redistribution of $O B$ star luminosity in the interstellar medium (Leisawitz and Hauser 1988, Ap. J., 332, 954). The model can be used as a diagnostic tool to probe the radiation field and matter density in an H II region, place constraints on the proximity and orientation of an illuminated molecular cloud with respect to the ionizing stars, test for the presence of small, transiently heated dust grains, and determine whether the dust-to-gas ratio is normal.

Predictions of the model agree qualitatively and quantitatively with observations of blister-type $H$ II regions ionized by well-studied OB clusters in which the distribution of dense neutral material is known. This is illustrated by a model for IRAS observations of the region around NGC 7380 (S142).

We plan to use the model in our survey of regions of massive star formation in the outer Galaxy to study OB stars embedded to various degrees in their parental molecular clouds.

On this poster, the model is illustrated in a series of figures. Here we model the environment of a $1.7 \times 10^{6} \mathrm{~L}_{\theta}$ cluster with $46 \%$ of the starlight radiated shortward of the Lyman limit. The ionizing (Lyc) photon production rate is $8.3 \times 10^{49} \mathrm{~s}^{-1}$. These stellar parameters are based on spectrophotometric obseryations of NGC 1893 (see Mermilliod 1976, Astr. Ap. Suppl., 24, 159) a cluster rich in early spectral type stars. The interstellar radiation field (ISRF) energy density is assumed to be the same as that in the solar neighborhood $\left(0.44 \mathrm{eV} \mathrm{cm}^{-3}\right)$. 


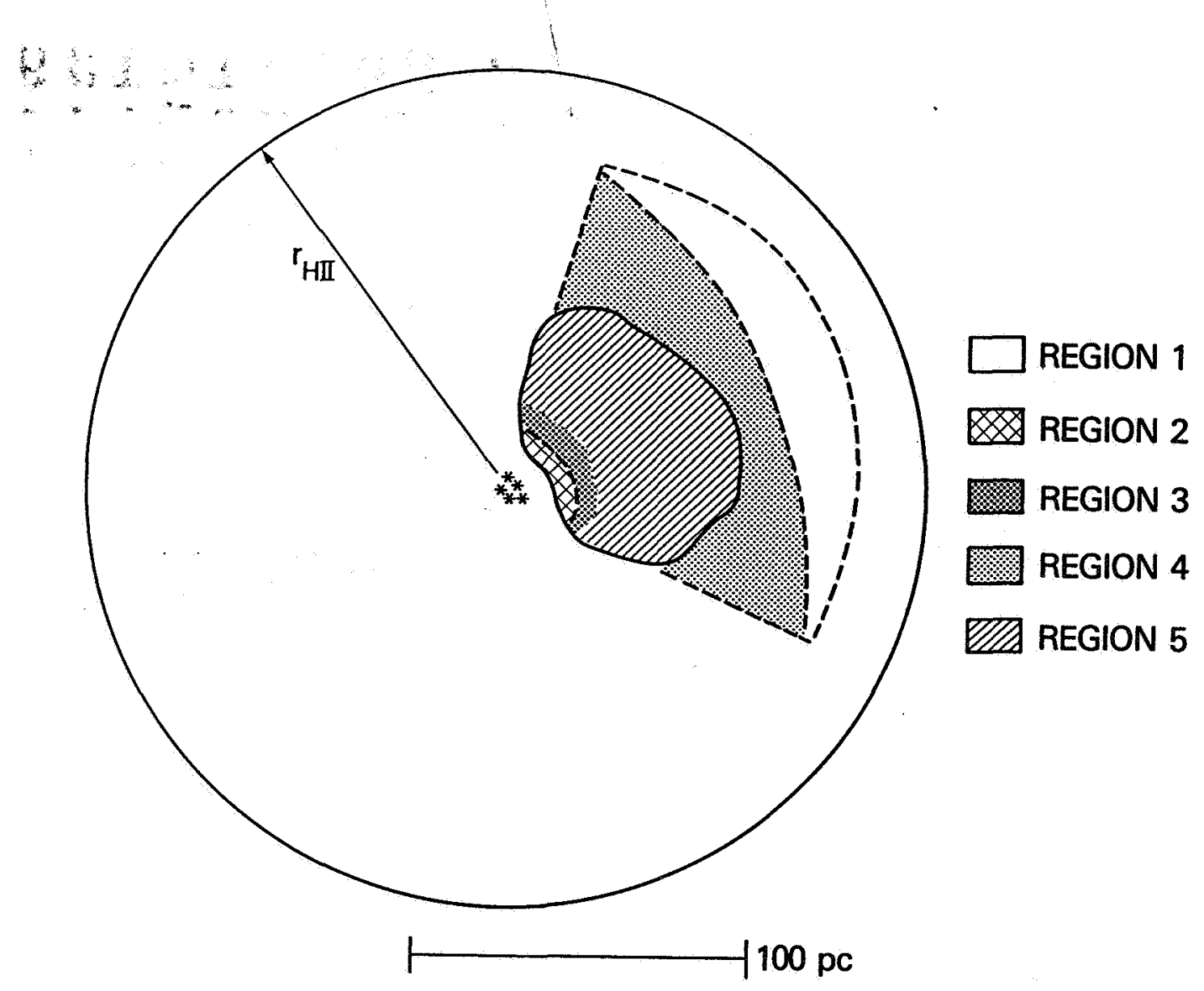

Figure 1: Elements of a typical model

The model has two components: a dense molecular cloud and a diffuse intercloud medium (ICM). Region 1 contains the ICM. Ionized gas is present in the $\mathrm{ICM}$ ( $\mathrm{n}=1 \mathrm{~cm}^{-3}$; Strömgren sphere radius, $\mathrm{R}_{\mathrm{S}}=130 \mathrm{pc}$ ) and in a $4.8 \mathrm{pc}$ thin layer (Region 2) on the illuminated surface of the nearby molecular cloud $\left(\mathrm{n}=30 \mathrm{~cm}^{-3}\right)$. Non-ionizing radiation from the central cluster penetrates the cloud to a greater depth (Region 3 ) and the entire cloud is heated externally by the ISRF. The molecular cloud surface is $6 \mathrm{pc}$ from the cluster and the thickness of the cloud is $30 \mathrm{pc}$. Because of direct absorption of some of the Lyc radiation by dust, the ICM is ionized to a radius $r_{H}$ II $=104 \mathrm{pc}$, somewhat smaller than $R_{S}$. The cloud is opaque at UV and visible wavelengths, so it casts a shadow in the ICM (Region 4) and its interior (Region 5) is relatively cold. Two radiative transfer calculations are done. The first is along a line from the cluster through Region 1; the second calculation starts at the cluster and follows a line through Regions 1, 2, 3, 5, and 4. 